\title{
Desempeño Ocupacional Realizante: una mirada a su trayectoria, aportes a su fundamentación teórica ${ }^{1}$
}

\author{
Fulfilling Occupational Performance: A look at its trajectory and \\ contributions to its theoretical foundation
}

\author{
Jenny Paola Castillo Aguas ${ }^{2}$ \\ Valeria Varón Caicedo ${ }^{3}$ \\ Recibido: 15 de febrero 2017 • Enviado para modificación: 30 de julio 2017 • Aceptado: 11 de agosto 2017 \\ Castillo, J. \& Varón, V. (2017). Desempeño Ocupacional Realizante: una mirada a su trayectoria, aportes a su \\ fundamentación teórica. Revista Ocupación Humana, 17 (1), 7-24
}

\begin{abstract}
RESUMEN
Se parte de una revisión de documentos que contemplan los postulados del primer modelo colombiano de Terapia Ocupacional, denominado Desempeño Ocupacional Realizante, y de investigaciones que lo han utilizado como base, para después identificar sus transformaciones a lo largo del tiempo y realizar algunos aportes. Se propone una nueva representación gráfica del Modelo que pretende explicar su dinámica permanente y bidireccional. Por último, se realiza una reflexión general de aspectos a tener en cuenta en su utilización y contribuir a su fortalecimiento. Se concluye que el Modelo de Desempeño Ocupacional Realizante ha facilitado y aportado a la formación académica y ha permitido generar reflexiones desde una perspectiva crítica a los profesionales de Terapia Ocupacional; así mismo, ha permitido profundizar en conceptos como la esencia espiritual y emocional, y cómo estas son partícipes en la productividad, socialización, autonomía y realización de cada persona o colectivo.
\end{abstract}

\section{PALABRAS CLAVE}

Conocimiento, terapia ocupacional

\section{ABSTRACT}

This article starts from a review of documents that contemplate the postulates of the first Colombian model of Occupational Therapy, called Fulfilling Occupational Performance, and some investigations that have used it as a foundation. Then, its transformations over time are

\footnotetext{
${ }^{1}$ Este artículo se deriva del trabajo de grado Prácticas y Reflexiones de Terapia Ocupacional que Aportan en la Fundamentación Teórica del Modelo Desempeño Ocupacional Realizante, realizado por las autoras para optar al título de terapeutas ocupacionales de la Universidad Nacional de Colombia y dirigido por la docente Lida Otilia Pérez Acevedo. Trabajo ganador del tercer puesto del Premio de Investigación Alicia Trujillo Rojas, decima versión (2016), en la categoría estudiantes

${ }^{2}$ Terapeuta Ocupacional. Terapistas del Country. Bogotá, Colombia.jpcastilloa@unal.edu.co

${ }^{3}$ Terapeuta Ocupacional. Fundación Niñez y Desarrollo. Bogotá, Colombia. vvaronc@unal.edu.co
} 
identified, and some contributions are proposed. We propose a new graphic representation of the Model that intends to explain its permanent and bidirectional dynamics. Finally, we present a general reflection of aspects to be taken into account in its use and to contribute to its strengthening. As a conclusion, we state that the Fulfilling Occupational Performance Model has facilitated and contributed to the academic formation, and it has allowed to generate reflections from a critical perspective to Occupational Therapy professionals. It has also allowed us to delve into concepts such as spiritual and emotional essence, and how these are part of productivity, socialization, autonomy and the fulfillment of each person and/or group.

\section{KEY WORKS}

Knowledge, occupational therapy

\section{Introducción}

Múltiples fueron las razones que dieron cabida a la creación del Modelo Desempeño Ocupacional Realizante, todas buscando comprender las necesidades y realidades que afrontaban para entonces el país y la Terapia Ocupacional (Trujillo, 1994a). Su construcción se ubica en el periodo comprendido entre 1982 y 1996, por parte de las terapeutas ocupacionales y docentes de la Universidad Nacional de Colombia Alicia Trujillo, Rosario Laserna, Laura Álvarez, Martha Torres y María Victoria Zapata. De acuerdo con sus autoras, los fundamentos del Modelo están ligados a una visión humanista, donde el ser humano está impulsado hacia la autorrealización por medio de la ocupación, la construcción de experiencias significativas y el actuar personal en ascenso o descenso (Trujillo,1995). Con el Modelo Desempeño Ocupacional Realizante se pretendía fortalecer los dominios de estudio de la profesión, además de crear bases sólidas para la formación de estudiantes y establecer los propósitos que permiten a la persona ser autónoma, productiva, influir en medios sociales y lograr su realización (Trujillo, 1994a). Desde entonces, fue clara la necesidad de reflexionar y aportar a sus fundamentos conceptuales, además de identificar aspectos que, desde el contexto colombiano, permitieran enriquecer sus componentes.

A inicios de 2014 surge desde un aula de clase la inquietud y la oportunidad de trabajar con y para el Modelo, tras identificar que los modelos son analogías del mundo real, representados en estructuras que simbolizan un evento o situación y permiten establecer un orden entre ellas, guiando el proceso de toma de decisiones dentro de la Terapia Ocupacional. Para 2015, se encontró que el Modelo tenía poca utilización en los escenarios prácticos, de manera que la recopilación de sus fundamentos teóricos podría facilitar ese uso, aportando a la centralización de conceptos, al entendimiento y comprensión del mismo dentro de la realidad actual del país.

Por ello, la investigación buscó conocer el recorrido del Modelo desde el inicio de su formulación hasta la 
actualidad, con el objetivo de identificar aportes teóricos y conceptuales relevantes, y provocar una reflexión que aporte a su fortalecimiento.

\section{Metodología}

La investigación se planteó desde el enfoque histórico hermenéutico y es de tipo documental. Se revisaron alrededor de cuarenta documentos incluyendo revistas, tesis, libros, documentos virtuales e impresos del periodo comprendido entre los años 1985 y 2015.

En estos documentos se rastrearon cronológicamente los cambios en la terminología, las descripciones, los postulados teóricos y las explicaciones graficas, así como las posturas de cada autor. Se hicieron cuadros comparativos para finalmente analizar, según nuestro criterio, los aportes a los que se podría llegar.

\section{Resultados}

\section{Recorridos del Modelo}

Entre los documentos revisados, cuatro dan cuenta de la evolución en la formulación del Modelo, a continuación se resumen los principales cambios y aportes identificados en cada uno de ellos.

\section{Terapia Ocupacional y Universi- dad, Modelo Desempeño Ocupa- cional y Realización Humana, su origen y alcances (Trujillo, 1994a).}

Este capítulo del libro Terapia Ocupacional y Universidad (Trujillo, 1994a) es el primero que expone el Modelo, para entonces denominado Desempeño Ocupacional y Realización Humana. Allí se define como una propuesta que simboliza el dominio de estudio de la Terapia Ocupacional, entendiéndolo como la relación existente entre los procesos ocupacionales (autocuidado, juego y trabajo, y sus componentes neuromotor, integrativo, sensorial, cognoscitivo, emocional y social), los procesos humanos (entendidos como niveles crecientes de subsistencia, socialización, dominio y actualización) y el medio ambiente (constituido por un espacio, una temporalidad, una estructura social económica e ideología que favorece o impide la realización). A partir de esa relación surgen tres subdominios: el primero, organización e integración, entendido como la recepción y asimilación de estímulos y la interpretación del mundo a través de vivencias organísmicas; el segundo, productividad, siendo la maestría de conocimientos, habilidades y destrezas, y el tercero, realización, visto como el trascender de los niveles de organización - integración y productividad hacia vivencias holísticas, que conlleva a la disposición personal hacia la expansión y la plenitud (Trujillo, 1994a).

Las autoras plantean cuatro postulados relacionados con la concepción del desempeño ocupacional del ser humano y la Terapia Ocupacional. El primero hace referencia al desempeño ocupacional, entendido como la transformación que se produce por la interacción significativa del hombre con el autocuidado, el juego y el trabajo en un contexto físico, social y temporal. El segundo expone cómo el desempeño ocupacional dinamiza la organización e integración, la productividad y 
la realización de la persona, teniendo en cuenta que estos tres subdominios influyen en el ascenso o el descenso del actuar ocupacional. El tercero indica que el terreno conceptual de la Terapia Ocupacional tiene que ver con el ascenso ocupacional del ser humano, con el estudio de los factores que lo debilitan o detienen, y con las condiciones requeridas para retomar la dinámica realizante. Se entiende por ascenso ocupacional aquellas características de la persona, influenciadas por un contexto y unas experiencias, que le permiten a ella o a un colectivo sentirse bien consigo misma y cumplir objetivos guiados por la motivación. El cuarto propone que el Modelo es una hipótesis o punto de partida dirigida a explicar el desempeño ocupacional a partir de los procesos humanos y los procesos ocupacionales contextualizados en el medio ambiente (Trujillo, 1994a).

Con base en estos postulados, las autoras proponen una representación gráfica del Modelo (Figura 1) donde se plantea un ascenso vertical y aparentemente rígido en la interacción entre los procesos ocupacionales y los procesos humanos, para lograr la realización humana a través del desempeño ocupacional.

Figura 1. Representación del Modelo Desempeño Ocupacional y Realización Humana, 1994.

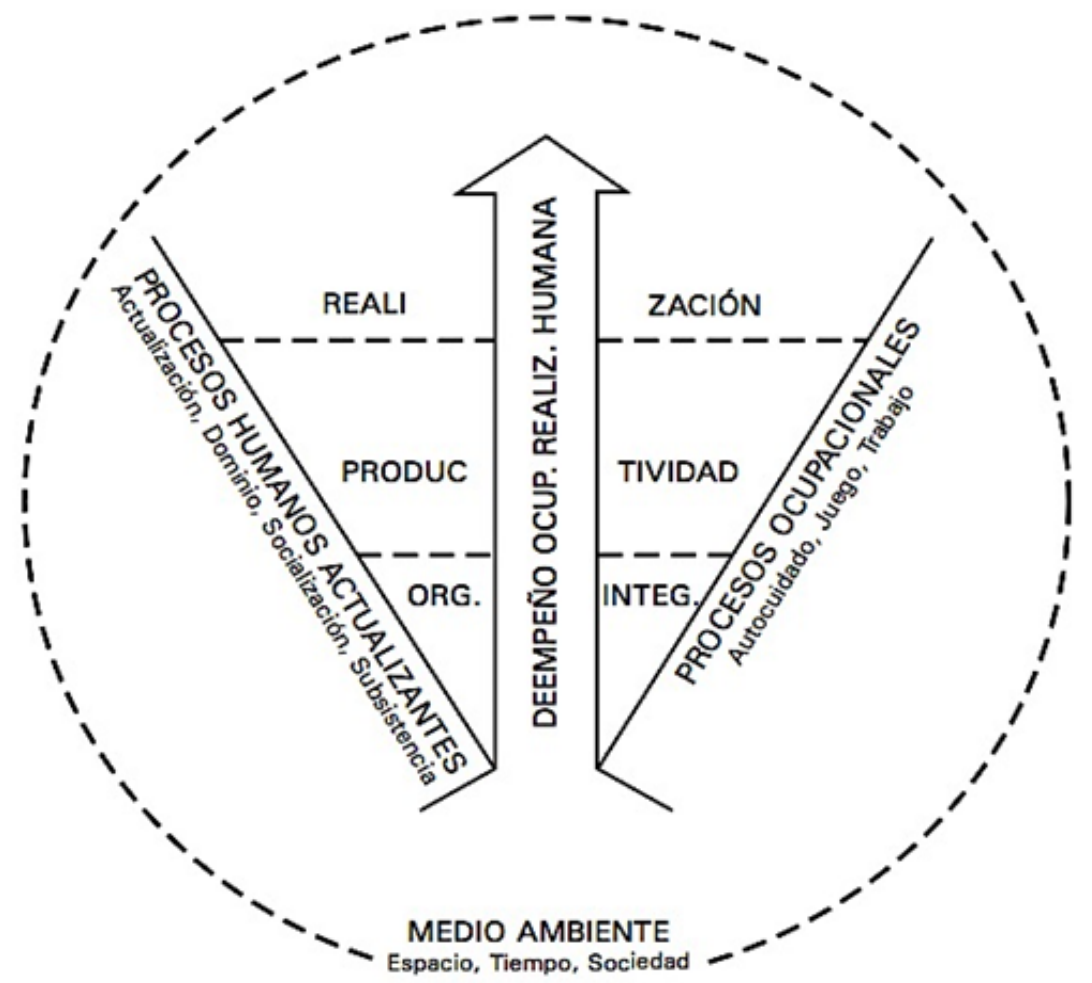

Fuente: Tomado de Trujillo, 1994a, p. 103. 
Desempeño Ocupacional Realizante: un modelo sobre los propósitos de la Terapia Ocupacional (Trujillo, 1995).

En este artículo, publicado en 1995 en la Revista Ocupación Humana, Alicia Trujillo presenta ante la comunidad profesional el Modelo Desempeño Ocupacional Realizante. La primera y más evidente transformación respecto al texto anterior está en el nombre, de Desempeño Ocupacional y Realización Humana a Desempeño Ocupacional Realizante; no se encontró en los documentos revisados un argumento que sustente este cambio.

En él se define el Modelo como una propuesta que aporta una visión humanista, simboliza el dominio de estudio de la Terapia Ocupacional y cuya estructura prioriza la direccionalidad de la profesión. Se enfatiza en los propósitos del actuar ocupacional de la persona, expresando en términos del movimiento ocupacional del ser humano un ascenso hacia la realización personal. El artículo mantiene que el desempeño ocupacional realizante surge de la interacción y confluencia de las tres dimensiones mencionadas en la versión de publicada en 1994, adicionando respecto a la primera dimensión, los procesos humanos, que estos permiten encontrar el significado en las experiencias y satisfacer las propias necesidades. En cuanto a la segunda dimensión, los procesos ocupacionales, se conciben las ocupaciones como proceso, ya que estas adquieren sentido en la medida que la persona les da significado y direccionalidad. Para la tercera dimensión, se retoma el término medio ambiente y se le da un valor fundamental en los programas de atención de Terapia Ocupacional; se menciona el contexto como el espacio físico en el cual se lleva a cabo la ocupación. Aquí es donde se considera a la cultura como componente esencial para el desempeño ocupacional realizante, porque la ocupación está inmersa y arraigada a la tradición y los valores de los grupos humanos, siendo los patrones o creencias culturales fuentes que estimulan o descalifican la realización individual (Trujillo, 1994a). La confluencia de tales propósitos genera un conjunto de experiencias que promueven la evolución de la persona hacia propósitos ascendentes, entendidos nuevamente como organización, integración, productividad y realización; en este texto, se reconoce que dichas experiencias están en permanente movimiento para llegar a una meta que es determinada por la persona (Trujillo, 1995).

La figura 2, empleada en este artículo para representar el Modelo, refleja el contexto temporal, social y espacial que influye en primera instancia en los procesos ocupacionales y los procesos humanos, los que a su vez repercuten en los propósitos de organización, integración, productividad y realización que apuntan al desempeño ocupacional realizante. Esta imagen se destaca por su forma circular, la cual representa una relación que puede entenderse como igualitaria, en tanto los componentes están en constante correlación y cambio.

Terapia Ocupacional: Conocimiento y práctica en Colombia (Trujillo, 2002).

Alicia Trujillo desarrolla en este libro aspectos pertinentes para la práctica de la profesión, entre ellos referentes 
Figura 2. Representación del Modelo Desempeño Ocupacional Realizante, 1995

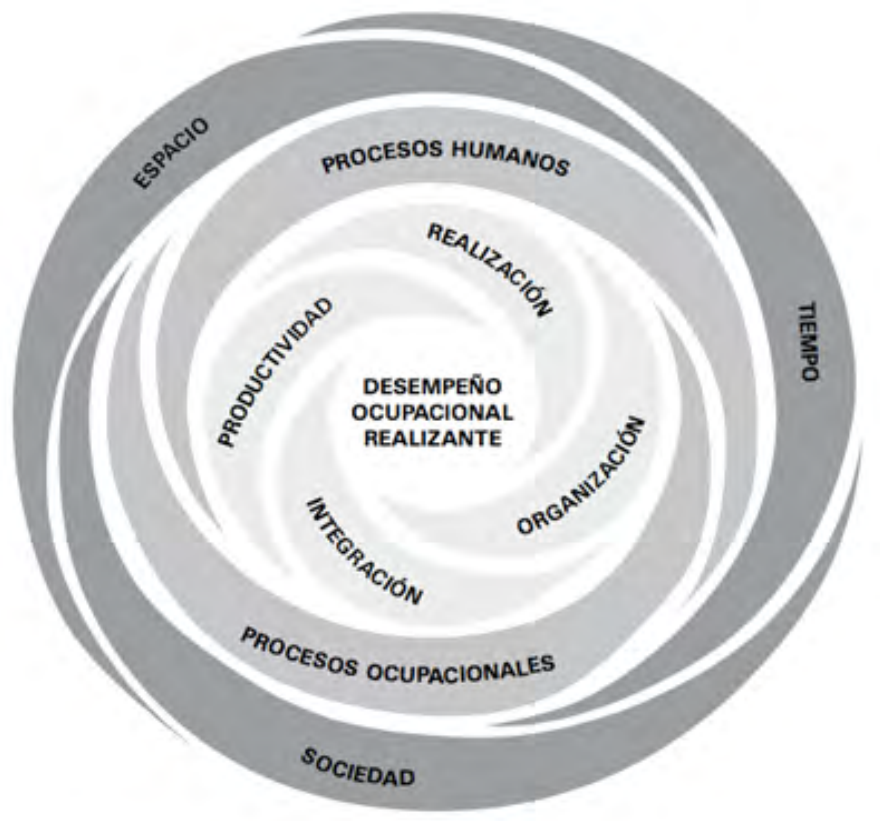

Fuente: Tomado de Trujillo, 1995, p. 12.

conceptuales como el Modelo Desempeño Ocupacional Realizante, al que define como una postura teórica de corriente humanista - existencial que pretende ubicar las diferentes concepciones teóricas de la profesión dentro de un marco de referencia en el cual el comportamiento humano pueda ser explicado (Trujillo, 2002). Así mismo, el texto postula que las personas, a través de su desempeño ocupacional (compuesto de características personales, ocupacionales y contextuales), tienden a su propia organización, integración, productividad y realización, siendo esta última el propósito más evolucionado y alto del desempeño ocupacional. Esta definición permite entender que la realización se logra al alcanzar el punto más alto de satisfacción en el desempeño ocupacional, gracias al cumplimiento de las demás categorías que propone el Modelo.

En la figura 3, incluida en el texto, la autora conjuga elementos del Modelo Desempeño Ocupacional Realizante con otros de la terminología uniforme de la Asociación Americana de Terapia Ocupacional - AOTA y de la Práctica Centrada en el Cliente de la Asociación Canadiense de Terapeutas Ocupacionales- $\mathrm{CAOT}^{4}$. Muestra una relación asociativa entre los componentes propios del Modelo (autonomía, socialización, productividad y realización, y desempe-

4. AOTA y CAOT, como se conoce a estas organizaciones por sus iniciales en inglés. 
Figura 3. Desempeño ocupacional: dominio de estudio de la Terapia Ocupacional

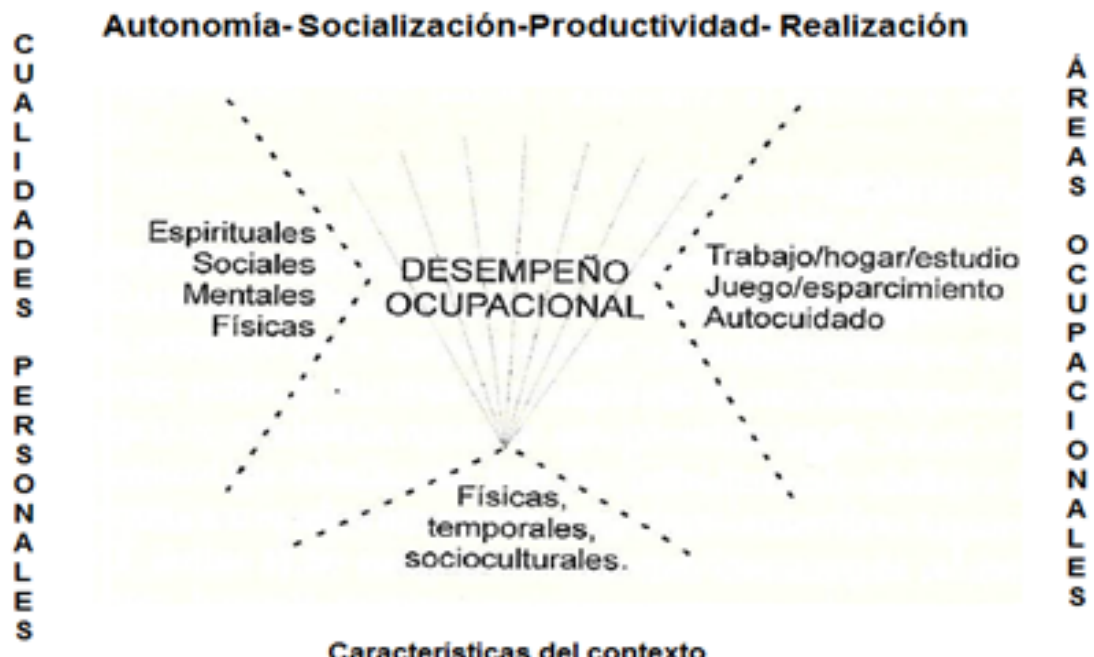

Fuente: Tomado de Trujillo, , 2002, p. 37.

ño ocupacional) y considera las características del contexto (físicas, temporales, socioculturales).

Con esta forma de representación gráfica, se entiende que las cualidades personales, las áreas ocupacionales y las características del contexto pueden relacionarse íntimamente entre sí de manera bidireccional y aportar a los propósitos ascendentes del desempeño ocupacional, sin necesidad de tener alguna jerarquía. En nuestra opinión, es necesario incluir algo que simbolice que la realización es un proceso dinámico que puede ir en ascenso o descenso según las ocupaciones, las cualidades personales y las particularidades del contexto físico, temporal y cultural.
Perspectivas de Terapia ocupacional frente al VIH/SIDA: una experiencia de VIHDA (Becerra \& Moreno, 2002, 2003).

Este texto es un trabajo de grado elaborado por Pilar Becerra y Maribel Moreno en el año 2002 para optar al título de terapeutas ocupacionales de la Universidad Nacional de Colombia, en el cual se realiza una propuesta práctica y conceptual para el abordaje de personas que viven con VIH/SIDA. Aquí se entiende el desempeño ocupacional realizante como una dinámica constante individual en la que se relacionan las variables: cualidad humana, desarrollo de las demandas ocupacionales, y características y oportunidades ofrecidas 
por el entorno; las cuales ayudan a construir los propósitos más importantes para las personas, relacionados con la autonomía y la productividad en los medios sociales, para lograr la realización.

En el texto se reflejan cambios en la taxonomía, pues se habla de cualidades humanas y demandas ocupacionales, en lugar de procesos humanos y procesos ocupacionales; también hay diferencias en los propósitos realizantes, no se hace referencia a organización e integración sino a autonomía y socialización. Se entiende autonomía como "la actuación libre e independiente de una persona que asume por sí misma su automantenimiento físico, emocional, espiritual intelectual, por medio de la realización de actividades significativas en forma eficiente, de acuerdo con sus capacidades e intereses, utilizando en forma creativa los recursos ambientales" (Becerra \& Moreno, 2002, p.126), y socialización, como un proceso de formación a través de la interacción con el contexto dirigido a la estabilidad personal (Becerra \& Moreno, 2002). Así mismo, se pasa del término medio ambiente al de contexto, que aunque incluye las características físicas, temporales y sociales planteadas desde la creación del Modelo, incluye además las caracteristicas familiares y culturales como parte de la historia personal y como un componente que brinda información y estímulos en respuesta a las necesidades de las personas ${ }^{5}$.

Becerra y Moreno (2002) realizan aportes propios en relación a los pos- tulados del Modelo, dirigidos a la influencia del contexto y a cómo las limitantes $\mathrm{u}$ oportunidades del mismo determinan la realización personal. Otro punto a resaltar es la importancia dada a la visión humanista, la cual permite un acercamiento a la realidad de las personas. Las autoras además incluyen variables como género, orientación sexual, clase y etnia como complemento del enfoque teórico, ya que tienen implicaciones en la calidad de vida, en el disfrute de la cotidianidad y en la tendencia a la realización de las personas. Otro aporte relevante de este trabajo es la creación de un instrumento de valoración propio del Modelo, basado en sus principios, desde una perspectiva dirigida a la población con VIH/SIDA, al cual se hace referencia más adelante.

En su trabajo, Becerra y Moreno (2002) toman como referencia un esquema elaborado en 2001 por el Grupo Ocupación y Realización Humana de la Universidad Nacional de Colombia, en el cual se involucran las dimensiones del Modelo Desempeño Ocupacional Realizante (Figura 4). En él, se quiere mostrar un dinamismo que no se logra del todo, ya que tanto en las cualidades como en las demandas no se visibiliza una interacción clara hacia el desempeño ocupacional realizante; por lo tanto, es pertinente pensar en una representación que permita reflejar la confluencia, la interacción y la direccionalidad de los componentes del Modelo.

\footnotetext{
${ }^{5}$ Becerra y Moreno toman estos conceptos del documento inédito “Definición Conceptual de los Componentes del Modelo Desempeño Ocupacional Realizante", elaborado en 2001 por el Grupo Ocupación y Relización Humana de la entonces Unidad de la Ocupación Humana de la Universidad Nacional de Colombia.
} 
Figura 4. . Modelo Desempeño Ocupacional Realizante, 2001

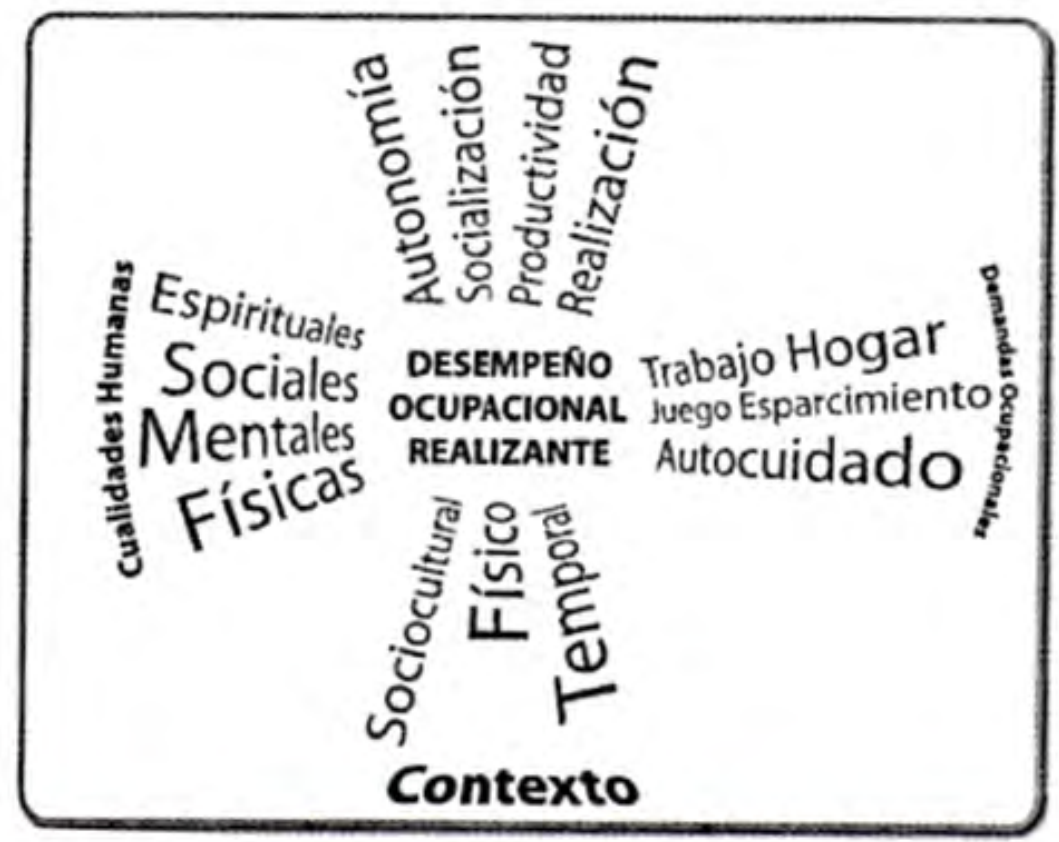

Fuente: Grupo Ocupación y realización Humana, 2001. Tomado de Becerra \& Moreno, 2003, p.30.

\section{Algunos usos académicos del Modelo Desempeño Ocupacional Realizante}

El Modelo Desempeño Ocupacional Realizante ha sido implementado en la academia desde diferentes frentes. A continuación se exponen de manera general tres de esos usos, así como los eventuales aportes que de ellos se han derivado para el Modelo.

\section{Modelo Desempeño Ocupacional del Escolar}

El Modelo Desempeño Ocupacional del Escolar (DOE), creado por Laura Álvarez en la década de los noventa, es una alternativa de re-contextualización del proceso formativo de terapeutas ocupacionales y un medio para dar vaIor al Modelo Desempeño Ocupacional Realizante (Álvarez, 2010). Desde este punto de vista, el DOE permite comprender cómo el ambiente demanda o facilita comportamientos ocupacionales y aporta a la capacidad de desempeño de las personas, aspectos relacionados con los postulados del Modelo Desempeño Ocupacional Realizante.

La figura 5 representa la relación existente entre los componentes del DOE. En el eje central se desarrolla la interacción entre las demandas escolares, las capacidades personales y el contexto, dirigida hacia el desempeño ocupacional del escolar e involucrando 
un accionar recíproco entre todos los agentes. Se intenta destacar que el resultado siempre es la modificación del estado de los diferentes elementos que lo componen, lo que significa que se basa en una interacción interpersonal, relacional y actitudinal que comprende a la persona como un ser social capaz de desarrollarse a través de la interacción con sus semejantes.

Figura 5. Modelo de Desempeño Ocupacional del Escolar, 2010.

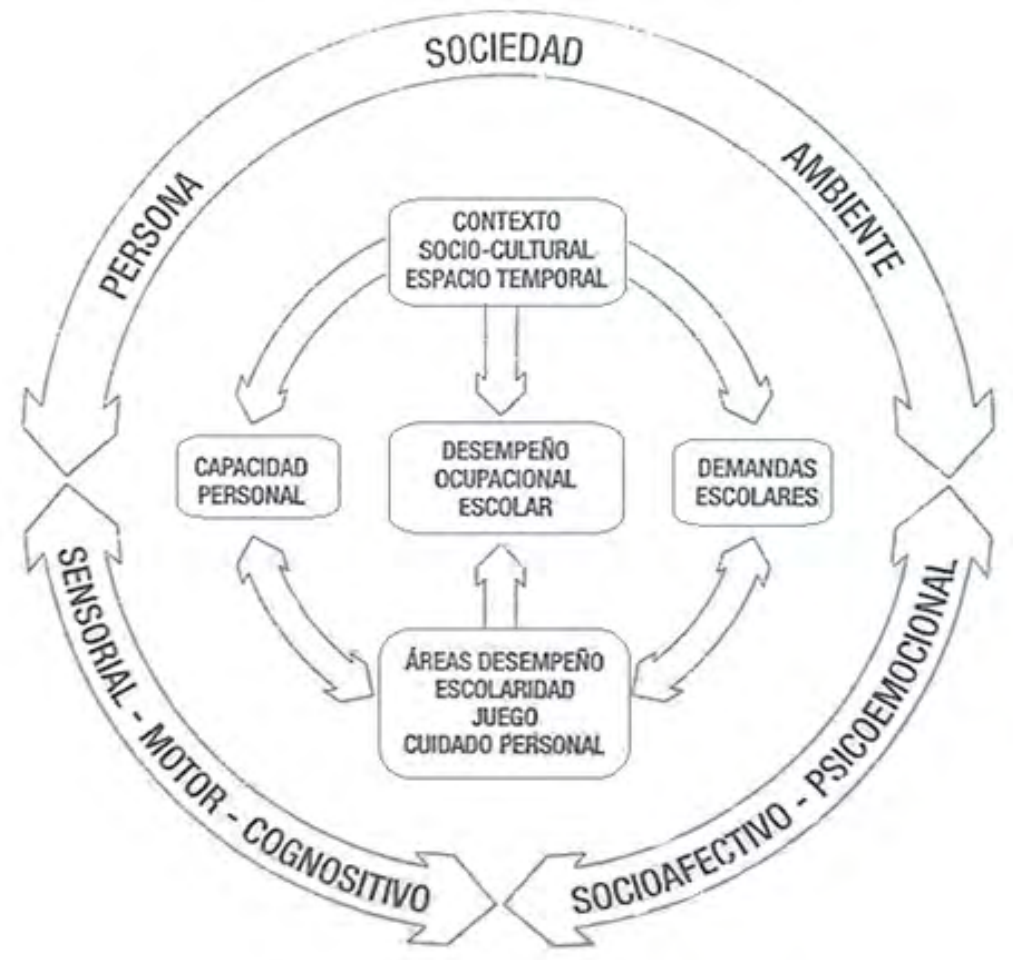

Fuente: Tomado de Álvarez, 2010, p. 164.

\section{Instrumento de valoración} del Desempeño Ocupacional Realizante

Como se ha mencionado, Becerra y Moreno (2002, 2003), a partir de una experiencia teórico-práctica, diseñaron un programa en el que implementaron los postulados del Modelo Desempe- ño Ocupacional Realizante. Para ello crearon un instrumento de evaluación orientado a estimar el estado de realización personal. El instrumento está conformado por un Manual de Aplicación y Calificación, el Formato de Valoración del Desempeño Ocupacional Realizante y el Formato del Perfil del Desempeño Ocupacional Realizante, y 
cuenta con validez aparente.

\section{Tipología de la noción de modelo} y su aplicación a dos modelos en Terapia Ocupacional

Esta tesis fue elaborada por la terapeuta ocupacional Jaqueline Cruz Perdomo para optar al título de Magíster en Filosofía en el año 2012; de ella se derivó el artículo titulado Análisis de dos modelos de Terapia Ocupacional a partir de la noción de modelo en la Filosofía de la Ciencia, que se encuentra publicado en la Revista Ocupación Humana (Cruz, 2012, 2015).

El Modelo Desempeño Ocupacional Realizante es abordado allí como medio para comprender las ideas de modelo y de teoría en las cuales se enmarcan los conocimientos en Terapia Ocupacional, concretamente para responder a la pregunta: ¿Qué tipo de modelo es el Modelo Desempeño Ocupacional Realizante? Frente a la cual, la autora realiza un análisis identificando sus características, en relación con diferentes nociones de modelo. En este sentido, Cruz $(2012,2015)$ resalta que el Modelo Desempeño Ocupacional Realizante cumple con la característica de ser una idealización, que se representa como una estructura simbólica formada por los elementos que lo componen; siendo la idealización un requerimiento del enfoque semántico de las teorías para la noción de modelo. Subraya además que aunque en Terapia Ocupacional los términos teoría y modelo se consideran diferentes, el Modelo Desempeño Ocupacional Realizante cumple las dos funciones, es decir, adicionalmente actúa como teoría acerca del Desempeño Ocupacional, ya que representa un aspecto de la realidad constituido por los elementos y conceptos que propone (Cruz, 2012, 2015).

\section{Discusión}

\section{El Desempeño Ocupacional Realizante como modelo y teoría}

El Modelo Desempeño Ocupacional Realizante ha sido identificado por las autoras revisadas como un modelo conceptual; a lo largo de sus 21 años de crecimiento, esta idea se mantiene. Pero, ¿qué entendemos por modelo conceptual? Desde nuestra reflexión e investigación ¿cómo entendemos el Modelo Desempeño Ocupacional Realizante? Al respecto, recurrimos a la definición que nos brinda Trujillo (2002):

...En la literatura de la Terapia Ocupacional se identifican diversos términos relacionados con el conocimiento científico, cuyo significado varía de autor a autor (Hagedorn, 1995; Kielhofner, 1994; Mosey, 1981; Reed, 1984). Estos términos son: marco de referencia, marco conceptual, marco teórico, modelo teórico, teoría y enfoque, entre otros. Todos ellos se diferencian por su amplitud, profundidad, complejidad, mensurabilidad y grado de utilidad conceptual o empírica... (p. 125)

A partir de lo anterior, resaltamos dos términos que consideramos importantes y que han sido retomados por Cruz $(2012,2015)$ : teoría y modelo. Según Trujillo (2002), el primero puede entenderse como un grupo de conceptos, definiciones y proposiciones inte- 
rrelacionadas que permiten organizar y categorizar, explicar o predecir los aspectos de la realidad. Podemos decir que el Modelo cumple con esas cualidades, ya que sus postulados y componentes pretenden representar un aspecto de la realidad, sea de una población específica o de una persona particular. Pero si bien el Modelo Desempeño Ocupacional Realizante, por lo dicho anteriormente, sería una teoría, esta noción aún no estaría suficientemente desarrollada, ya que la interacción entre los conceptos, la importancia del contexto, cómo se entienden los procesos ocupacionales y los humanos, y su influencia en los propósitos, aún no son suficientemente claros, lo que hace difícil entender la dinámica que el Modelo quiere plasmar.

Ahora bien, el término modelo, el cual Trujillo (1994b) define como la representación de un evento o situación que le permite a la persona ilustrar ideas y establecer órdenes entre ellas, además de mostrarlas de manera gráfica, pictórica, esquemática o matemática; además "guía y orienta el proceso de toma de decisiones, porque al ser una analogía del mundo real, permite clarificar hechos complejos, analizar relaciones, pronosticar posibles desempeños y prever las consecuencias de las medidas que se adopten" (Trujillo, citado por Cruz, 2012, p.34). Con esto podemos entender que el Desempeño Ocupacional Realizante también es un modelo, ya que su estructura es simbólica y está formada por los procesos humanos, los procesos ocupacionales, el contexto y los propósitos ocupacionales; con la ayuda de la representación gráfica, busca plasmar el dinamismo de los componentes y logra que dicha definición sea adecuada.
En este punto y con la anterior explicación, continuamos hacia el entendimiento de lo que es un modelo conceptual. Para ello nos remitimos a Kielhofner (citado por Cruz, 2012), quien indica que "...un modelo conceptual de la práctica puede definirse como un conjunto de argumentos teóricos en evolución que se traducen en tecnología específica para la práctica y que son perfeccionados y evaluados a través de la investigación" (p.34). Para este autor, un modelo conceptual se constituye a partir de un conocimiento interdisciplinar que articula la teoría para dar una explicación a los fenómenos particulares; brinda herramientas, procesamientos o materiales para la aplicación terapéutica, y se comprueba a través de la investigación. Estas características hacen parte del Desempeño Ocupacional Realizante, dado que el Modelo está formado por una base teórica interdisciplinar desde la Biología, la Psicología, la Antropología y fundamentos culturales, los cuales son esenciales para comprender la dinámica relacional entre la persona, la ocupación y el contexto (Cruz, 2012); además, representa fenómenos guiados al desempeño ocupacional. No obstante, en la actualidad el desarrollo tecnológico del Modelo presenta importantes restricciones, dado que no provee el suficiente despliegue operativo como instrumentos de evaluación o herramientas que permitan la recolección de información, siendo este un punto que limita su potencia.

Con todo lo anterior, podemos dar respuesta al primer interrogante diciendo que el Modelo Desempeño Ocupacional Realizante, por su estructura, postulados y fundamentos, es un modelo conceptual que sirve como guía para 
el actuar de la Terapia Ocupacional. Por otro lado, que para seguir fortaleciendo dicha nominación es necesario que los y las terapeutas ocupacionales hagan uso de él en la academia y en la vida profesional práctica, en la atención de las poblaciones colombianas y en los procesos investigativos.

\section{Conceptos básicos del Modelo: aportes y reflexiones}

\section{Procesos Humanos}

Consideramos necesario seguir manejando el término "proceso", teniendo en cuenta la connotación dinámica que ello representa. Como lo menciona Trujillo (1995), citando a Jantsch, proceso implica movimiento, transformación, y transición en celeridad y dirección, lo que está en sintonía con la representación que pretende dar el Modelo de la interacción entre todos los componentes y su influencia en la persona y su contexto.

Retomamos los procesos humanos como aquella interacción entre las características inherentes de la persona -tales como sus cualidades físicas, mentales, emocionales, espirituales y sociales-, que permiten encontrar significado en las experiencias y así mismo satisfacer necesidades personales y colectivas. Cuando existe una alteración en cualquiera de estas características, el desempeño ocupacional realizante se puede ver afectado dado el dinamismo del actuar ocupacional y la conjugación de procesos humanos, procesos ocupacionales y propósitos ocupacionales. Sin embargo, esta alteración está condicionada por el contexto, ya que de este depende que la persona logre tomar medidas que fa- vorezcan su desempeño ocupacional hacia la realización.

\section{Procesos ocupacionales}

Para esta dimensión, consideramos que el término adecuado y que se acopla a las características del modelo es proceso. Esto porque además indica un trascurrir dinámico de las ocupaciones y de los diferentes componentes inmersos en el desempeño ocupacional realizante.

El autocuidado contempla las rutinas y hábitos básicos para alimentación, vestido, higiene, cuidado personal y desplazamiento. El juego/esparcimiento está relacionado con las actividades lúdicas en la infancia o el esparcimiento en la vida adulta. El proceso ocupacional trabajo comprende tanto la actividad laboral como el mantenimiento del hogar y el estudio; trabajo y estudio pueden desarrollarse al mismo tiempo en la edad adulta, por lo que es necesario abordarlos de manera independiente pues su dinámica puede influir de formas distintas en el desempeño ocupacional de la persona. Los tres procesos conllevan demandas sensoriomotoras, cognoscitivas y socioafectivas (Trujillo, 1995).

\section{Contexto}

A nuestro modo de entender, es el componente con mayor peso en lo que respecta a la realización personal, ya que de él surgen diferentes variables que determinan el actuar ocupacional. Puede influir de manera directa en el desempeño ocupacional, es decir, el contexto aprueba, facilita o legitima el desempeño, o a la inversa, lo limita, restringe o descalifica (Trujillo, 2002). 
Los elementos del contexto, según Trujillo (2002), están clasificados en características físicas, temporales y socioculturales, cada una con una importancia especial en la dinámica del Desempeño Ocupacional Realizante, dado que posibilitan y particularizan el actuar personal y colectivo, teniendo en cuenta aspectos estructurales, cronológicos y culturales; estos últimos, fundamentales para entender el desempeño ocupacional en un país como Colombia, donde la diversidad y la multiculturalidad son tan marcadas.

\section{Propósitos Ocupacionales}

OrgANIZACIÓN / aUtONOMÍA. La organización está relacionada con las cualidades personales que posibilitan el desempeño ocupacional y consisten en la disposición que tiene la persona para atender a estímulos del medio ambiente (Trujillo, 1995). La autonomía es entendida como la condición de la persona de actuar por sí mismo, de manera independiente, según las metas y siguiendo las normas establecidas por el grupos social al que pertenece (Trujillo, 2002). Con esto podemos inferir que la organización puede tener un carácter más colectivo y social que la autonomía, ya que esta última alude a la persona como tal en lo que se refiere a la toma de decisiones. Sin embargo, desde nuestra perspectiva la autonomía puede ser vista desde un enfoque social, no netamente individual; acorde a lo dicho por Gough (2007/08), quien expone que la misma está determinada por el grado de comprensión cultural y el rango de oportunidades. De esta manera, definimos autonomía como la condición personal y social de actuar, expresar, pensar y participar de manera libre dentro de un contexto físico, social y cultural que permita satisfacer necesidades personales-sociales relacionadas con el cumplimiento de una meta, y que de igual manera permita ofrecer experiencias de intercambio social.

INTEGRACIÓN / SOCIALIZACIÓN. La integración, según Trujillo (1995), es la manera como la persona relaciona hechos, se apropia del significado de las experiencias ocupacionales y las utiliza como aprendizaje en circunstancias similares. La socialización, entre tanto, es la condición de los seres humanos que los motiva a interactuar e intercambiar con otros para favorecer el cumplimiento de sus necesidades de subsistencia y sociales (Trujillo, 2002). Creemos necesario resaltar tres aspectos a tener en cuenta en este propósito: la experiencia, el mecanismo relacional y la condición social, para entender que la organización y la integración se complementan, de manera que el desempeño ocupacional construido con las experiencias permitirá a las personas y comunidades actuar socialmente para suplir las necesidades antes mencionadas. Consideramos que el término que mejor engloba el significado de este propósito es socialización; pues aunque la palabra integración hace más evidente la conjugación de experiencia, conocimiento y socialización, en nuestra opinión el propósito va más allá de reunir estas características, su objetivo es agrupar factores necesarios para la socialización.

Productividad. Trujillo, Álvarez, Torres y Zapata (citadas por Trujillo, 1995) definen la productividad como la maestría de conocimientos, habilidades y destrezas que permite a la persona cumplir con tareas de utilidad individual y social. Por otro lado, Becerra y Moreno 
(2002) indican que es el resultado de acciones eficientes y eficaces que desarrolla la persona con un propósito, sea físico, social, espiritual, económico o emocional y que proporciona diferentes grados de satisfacción. No obstante, creemos en otros tipos de productividad que no transitan por ahí. En términos del Desempeño Ocupacional Realizante, los procesos y propósitos de cada persona tienen una dinámica particular, por eso queremos resaltar, más que la utilidad, el significado que cada quien le da a su actuar en un contexto. Así, el propósito de productividad debe trascender y guiar nuestro actuar ocupacional, no en términos de utilidad sino de grado de significancia, que tanto a nivel personal como colectivo aporta a construir un desempeño ocupacional realizante.

REALIZACIÓN. Este propósito ha mantenido su nombre y definición hasta la actualidad. Trujillo (1994a, 1995, 2002) entiende la realización como un trascender de los otros propósitos hacia vivencias holísticas, lo que lleva la disposición personal hacia la expansión y la plenitud. Becerra y Moreno (2002) señalan que la realización es un "horizonte al que aspira llegar toda persona más allá de la satisfacción; es una disposición hacia las vivencias de expansión y plenitud que se expresan en la madurez de las dificultades humanas y en la armonía del ser..." (p. 126). Con estas dos concepciones nos surgen varias reflexiones; en primer lugar, debemos mostrar que el trascender de los propósitos de autonomía, socialización y productividad no necesariamente se dan al mismo tiempo. Es decir, en determinado momento una persona o comunidad puede lograr la plenitud dado que alcanza su propósito de autonomía; aunque en lo productivo no ocurra lo mismo, aun así llega al estado de expansión nombrado como realización. También es posible que todos los propósitos esten equilibrados en plenitud, lo que igualmente llevaría a la realización.

\section{Propuesta gráfica para el Modelo}

Sin desconocer los esfuerzos previos, presentamos una propuesta gráfica para el Modelo Desempeño Ocupacional Realizante (Figura 6).

El gráfico muestra el Desempeño Ocupacional Realizante desde dos perspectivas. La parte A indica la interacción dinámica de los seres humanos con el contexto, este nos puede brindar posibilidades o estar en relación solidaria permanente, lo que se representa a partir de las líneas punteadas; por el contario, también puede limitar u obstaculizar las interacciones y oportunidades que permiten tener experiencias realizantes. La parte B representa la dinámica en las personas, refleja la direccionalidad en cada una de las interacciones, así como las fluctuaciones de los propósitos ocupacionales, representadas con resortes, influyendo en el desempeño ocupacional.

Desde el exterior hacia el centro, el gráfico inicia con un hexágono que representa el contexto en donde la persona está inmersa, el cual comprende agentes sociales, temporales, culturales y espaciales, los cuales influyen en los procesos humanos, los procesos ocupacionales y en la confluencia de los propósitos ocupacionales. Su forma obedece a que, según Talero (2015), el contexto puede interpretarse como un panal de abejas en el que cada perso- 
na tiene su propio hexágono y puede encajar perfectamente en otro, es decir, sin importar el lugar en el que se encuentre, su cultura está arraigada a ella. Sin embargo, pueden existir modificaciones a lo largo de la vida, y a su vez aportar en los procesos de otras personas o colectivos, por lo que se traza de forma punteada.

Luego se observa un círculo conformado por dos flechas unidas entre sí, indicando que los procesos humanos y ocupacionales están en relación constante y recíproca. De la misma manera, estos se relacionan con los propósitos ocupacionales, es decir, explica que los componentes no pueden ser tomados de forma aislada, ya que existe una mutua influencia entre ellos que puede limitar o aportar al desempeño ocupacional realizante. Finalmente, en el centro se encuentra el desempeño ocupacional unido al resto de sus componentes por medio de espirales con flechas en ambos sentidos, las cuales representan que la confluencia de todo lo anterior lleva a momentos ascendentes y descendentes.

Figura 6. Representación propuesta para el Modelo Desempeño Ocupacional Realizante, 2015.

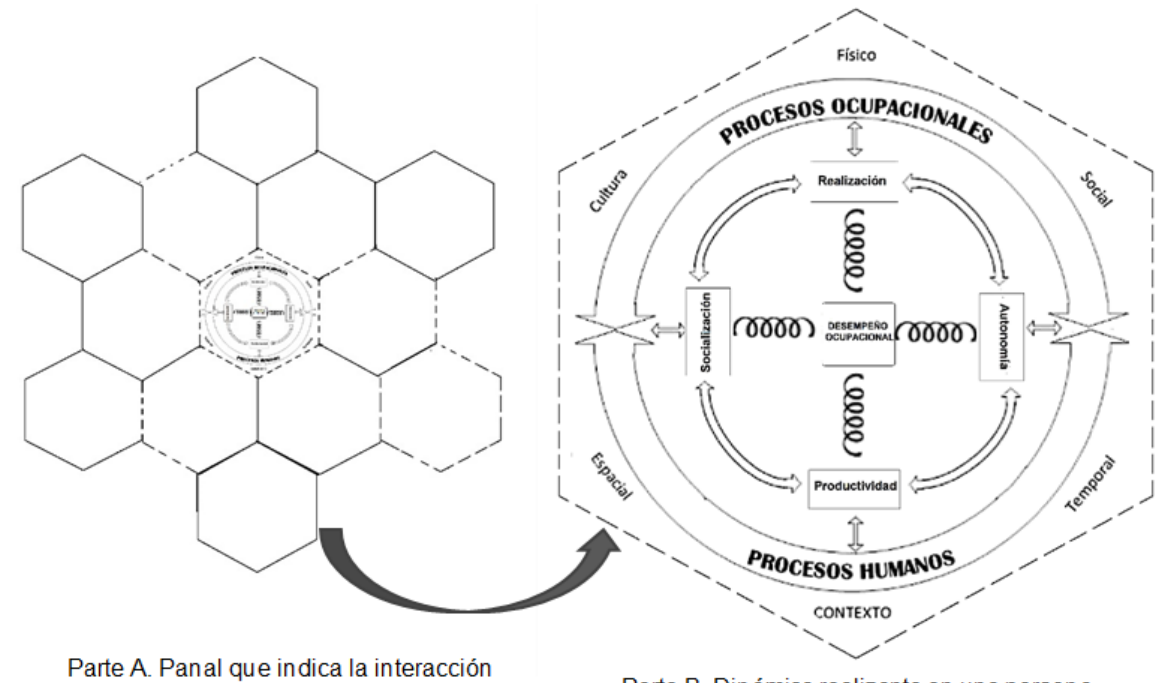
constante con el contexto.

Parte B. Dinámica realizante en una persona.

Fuente: Castillo \& Varón, $2015^{6 .}$

${ }^{6}$ Elaboración propia de las autoras, con la colaboración de Lida Pérez y Wendy Vargas. 


\section{Conclusiones}

El Modelo Desempeño Ocupacional Realizante es una herramienta creada hace poco más de veinte años, su implementación y fortalecimiento han sido escasos. El Modelo ha facilitado y aportado a la formación académica, ha permitido la generación de reflexiones y la formación de profesionales con posturas críticas que intentan posicionar la profesión y entender la importancia de la persona, su contexto y su realización como eje de la intervención, no solo como un ser partícipe del sistema. Consideramos necesario rescatar el trabajo realizado por sus autoras, contemplando que es el primer modelo colombiano propio de Terapia Ocupacional, y que por tanto debería mantenerse en discusión y actualización constantes.

Como modelo conceptual, el Desempeño Ocupacional Realizante facilita explicar la realidad de una persona, sin embargo, requiere ser fortalecido a través de herramientas de evaluación que promuevan la puesta en práctica de sus postulados. Es necesario continuar aplicando y adaptando el instrumento de evaluación existente para comprobar su fiabilidad y potenciar su uso, así como crear otros nuevos que permitan ampliar la perspectiva humanista desde una visión no solo personal si no colectiva, y así enriquecer el accionar profesional.

La investigación nos permitió identificar la necesidad de profundizar en conceptos del Modelo como la esencia espiritual y emocional de las personas y las comunidades, y en cómo estas influyen en la productividad (entendida como grado de significancia y valor social y personal), la socialización, la autonomía y la realización.

Por otro lado, identificamos al contexto cultural como eje principal en la comprensión de las personas y los colectivos desde Terapia Ocupacional, ya que a partir de sus posibilidades o limitaciones puede influenciarse el desempeño de manera directa. Por ello, es pertinente dar mayor valor a las cualidades culturales, partiendo de la realidad colombiana, donde la diversidad que caracteriza las diferentes regiones es una fuente de riqueza en la concepción de la realización.

Por último, la propuesta gráfica para el Modelo es un aporte a la representación de la interacción dinámica y compleja entre los elementos que lo componen, que queda así a disposición de la comunidad académica y profesional.

\section{Referencias}

Álvarez, L. (2010). Terapia Ocupacional en educación, un enfoque sensorial en la escuela. Bogotá: Universidad Nacional de Colombia.

Becerra, P. \& Moreno, M. (2002). Perspectivas de Terapia ocupacional frente al VIH/SIDA: una experiencia de VIHDA. (Tesis de pregrado). Universidad Nacional de Colombia, Bogotá.

Becerra, P. \& Moreno, M. (2003). Perspectivas de Terapia ocupacional frente al VIH/SIDA: una experiencia de VIHDA. Revista Ocupación Humana, 10(1,2), 27-39

Castillo, J. \& Varón, V. (2015). Prácticas y reflexiones de Terapia Ocupacional, 
que aportan a la fundamentación teórica del modelo del Desempeño Ocupacional Realizante. (Tesis de pregrado). Universidad Nacional de Colombia, Bogotá.

Cruz, J. (2012). Tipología de la noción de modelo en ciencia y su aplicación a dos modelos en Terapia Ocupacional. (Tesis de Maestría). Universidad del Valle, Cali.

Cruz, J. (2015). Análisis de dos modelos de Terapia Ocupacional a partir de la noción de modelo en la Filosofía de la Ciencia. Revista Ocupación Humana, 15 (1), 35 - 47.

Gouh, I. (2007/08). El enfoque de las capacidades de M. Nussbaum: Un análisis comparado con nuestra teoría de las necesidades humanas (Traductores $\mathrm{Na}$ gore, L. \& Silva, S). Papeles de relaciones ecosociales y cambio global, 100, 177-202.

Talero, P. (2015). Culturally responsive care in occupational therapy: an entry-level educational model embedded in service-learning. Scandinavian Journal of Occupational Therapy, 22, 95-102.
Trujillo, A. (1994a). Modelo de desempeño ocupacional y realización humana: su origen y alcance. En A. Trujillo (Ed.) Terapia ocupacional y Universidad (pp. 99-112). Bogotá: Universidad Nacional de Colombia.

Trujillo, A. (1994b). Diseño de modelos en Terapia Ocupacional. En A. Trujillo (Ed.) Terapia ocupacional y Universidad (pp. 91-97). Bogotá: Universidad Nacional de Colombia.

Trujillo, A. (1995). Desempeño Ocupacional Realizante: un modelo sobre los propósitos de la Terapia Ocupacional. Revista Ocupación Humana, 6 (2), 11 21.

Trujillo, A. (2002). Terapia Ocupacional. Conocimiento y práctica en Colombia. Bogotá: Universidad Nacional de Colombia. 